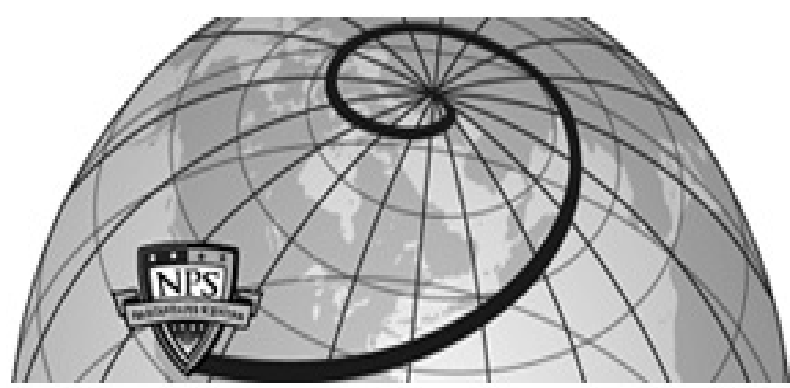

Calhoun: The NPS Institutional Archive DSpace Repository

Rest-to-Rest Slew Maneuver of Three-Axis Rotational Flexible Spacecraft

Kim, J.J.; Agrawal, B.N.

17th IFAC World Congress, July 6-11, Seoul, Korea, 2008.

https://hdl.handle.net/10945/34522

This publication is a work of the U.S. Government as definedin Title 17, United States Code, Section 101. As such, it is in thepublic domain, and under the provisions of Title 17, United StatesCode, Section 105, is not copyrighted in the U.S.

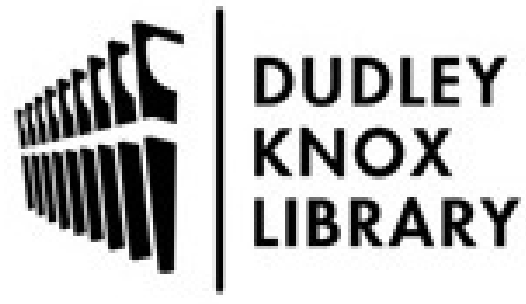

http://www.nps.edu/library
Calhoun is the Naval Postgraduate School's public access digital repository for research materials and institutional publications created by the NPS community. Calhoun is named for Professor of Mathematics Guy K. Calhoun, NPS's first appointed -- and published -- scholarly author.

Dudley Knox Library / Naval Postgraduate School 411 Dyer Road / 1 University Circle Monterey, California USA 93943 


\title{
REST-TO-REST SLEW MANEUVER OF THREE-AXIS ROTATIONAL FLEXIBLE SPACECRAFT
}

\author{
Jae Jun Kim $^{1}$ and Brij N. Agrawal ${ }^{2}$ \\ Naval Postgraduate School, Monterey, CA 93943
}

\begin{abstract}
This paper presents a slew maneuver control design of three-axis rotational flexible spacecraft. The focus of the work is to investigate the nonlinear effect of the three axis maneuver for a flexible spacecraft when a vibration suppression technique for linear systems such as input shaping is used in the control design. A simple method of slewing three-axis rotational spacecraft using input shaping is proposed and the proposed technique is implemented on an experimental three-axis spacecraft simulator.
\end{abstract}

Keywords: High Accuracy Pointing, Guidance, Navigation and Control of Vehicles

\section{INTRODUCTION}

For spacecraft applications where rapid slew maneuvers for the acquisition of a target point are required, the interaction of flexible structures with the spacecraft attitude control system can significantly degrade performance in terms of pointing accuracy and acquisition time. With the current trend in spacecraft design of increased flexibility, multiple bodies, and stringent attitude control accuracy requirements, conventional control techniques may not be adequate to meet performance requirements of future spacecraft missions. Developing controls for flexible spacecraft is an especially challenging task. For point-to-point slew maneuvers for Line of Sight (LOS) spacecraft, the residual vibration at the end of the maneuver significantly degrades the pointing accuracy. This residual vibration also increases slewing time especially for spacecraft with large structures and low frequency modes. To properly design a slew maneuver control system, pre-planned feedforward control profiles and corresponding reference trajectories are required. Especially for fast slew maneuvers of a flexible spacecraft, careful planning of slew trajectories and feedforward control input is a crucial element of the control design. The slew trajectories and feedforward control design is based on the mathematical model of a spacecraft. Therefore, accurate modelling of a spacecraft is also important. Traditionally, spacecraft are modelled as rigid bodies, and any vibration resulting from the flexible structures are considered to be disturbances. Therefore, traditional feedforward control is of the smooth bang-bang type, where rapid slew is obtained from the nature of the bang-bang control while the disturbance from the flexible structure excitation is reduced by smoothing the edges of the bang-bang control. It is generally known that there is a trade-off between the control actuation time and the level

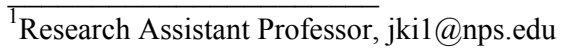

${ }^{2}$ Distinguished Professor, agrawal@nps.edu of flexible structure excitations with this approach. Therefore, it may require unnecessarily large control actuation time to obtain the required level of vibration suppression. Instead of reducing structural excitations with the smooth control profile, the input shaping technique attempts to cancel structural vibrations by convoluting a reference input with an impulse sequence. With the exact knowledge of the system frequencies, targeted structural frequencies at the end of the maneuver can be completely eliminated in a finite time. The input shaping technique was developed for linear systems and therefore, direct application to the three axis rotational flexible spacecraft problem is somewhat limited. For spacecraft problems with slow maneuvers, the non-linear coupling between three axes is relatively weak. Much previous work has utilized either linearization or small angular rate assumptions to apply input shaping techniques. Furthermore, the linear model can be decoupled (Eigen-axis rotation) and written in modal coordinates, which permits us to examine it as a single-axis rotational spacecraft problem. In this paper, we attempt to include a nonlinear coupling term between the three axes in the design of the reference feedforward control profiles. Input shaping technique is also redesigned for this coupled three-axis rotational spacecraft problem. The proposed technique is implemented on the experimental three-axis rotational spacecraft test-bed.

\section{BACKGROUND}

A rigid body spacecraft can be represented in the spacecraft body coordinates as

$$
J \dot{\omega}+\omega \times J \omega=B u
$$

where $J$ is a inertia matrix, $\omega$ is a angular rate, $u$ is a control input, and $B$ is a control influence matrix. The cross product term in Equation 1 appears nonlinearly in the equation. For slew maneuvers with large angular rates during the maneuver, effect of the nonlinear term may become significant. Therefore, it may be useful to include the non-linear effect when designing control profiles for 
three-axis rotational spacecraft. A method to determine control profiles including the nonlinear coupling for rigid body spacecraft is previously proposed by Bell and Junkins (1994). The idea is to first design slew trajectories using linear model, then design a control profile which follow the trajectories. The nonlinear coupling is included when the control profile is determined. For designing slew trajectories as a first step, simple uncoupled linear model is used. The equation of motion of three-axis uncoupled rigid bodies is written as

$$
J \ddot{\theta}=B u
$$

where $J$ is a diagonal inertia matrix, $B$ is a diagonal control influence matrix, and $\theta$ represents the state of the reference trajectory in the global coordinates.

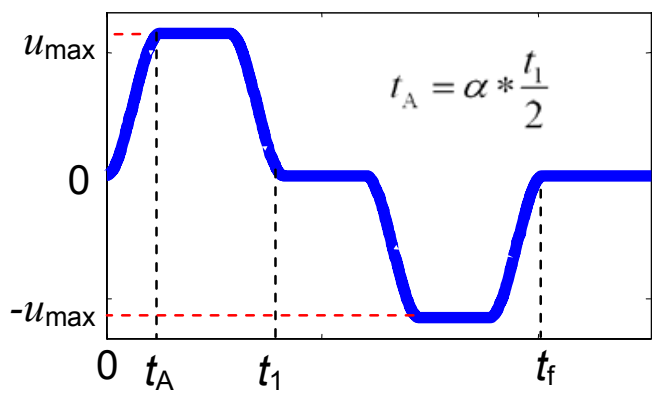

Figure 1: Smooth Bang-off-Bang Profile Example

The control input can be designed to meet the slew angle required separately for each axis, which can be written as

$$
u=u_{\max } f\left(t, t_{f}, \alpha\right)
$$

where $u_{\max }$ is the maximum available control for control design and $f\left(t, t_{f}, \alpha\right)$ is a function typically represent a smooth bang-off-bang type control as shown in Figure 1. The off period is due to momentum saturation (for momentum transfer actuators) or fuel consumption constraints (for jet thrustering actuators). This smooth bangoff-bang is a function of smoothing parameter $(\alpha)$ the final time $\left(t_{f}\right)$. It is noted that the final feedforward control input may be larger than $u_{\max }$ because the final control input is designed to follow the trajectories designed with assumed uncoupled linear model. Therefore, there should be some margin between maximum control input for control design and the actual maximum control input with this approach.

With the control input shown in Equation 3, the reference angle and angular rate trajectories in the global coordinates for the uncoupled equation of motion become

$$
\begin{aligned}
& \ddot{\theta}_{r e f}(t)=J^{-1} B u_{\max } f\left(t, t_{f}, \alpha\right) \\
& \dot{\theta}_{r e f}(t)=\dot{\theta}_{0}+J^{-1} B u_{\max } \int_{0}^{t} f\left(\tau, t_{f}, \alpha\right) d \tau \\
& \theta_{\text {ref }}(t)=\theta_{0}+\dot{\theta}_{0} t+J^{-1} B u_{\max } \int_{0}^{t} \int_{0}^{\tau} f\left(\eta, t_{f}, \alpha\right) d \eta d \tau
\end{aligned}
$$

For rest-to-rest maneuvers, the total maneuver angle becomes

$$
\theta_{f}-\theta_{0}=J^{-1} B u_{\max } \int_{0}^{t_{f}} \int_{0}^{\tau} f\left(\eta, t_{f}, \alpha\right) d \eta d \tau
$$

The Equation 5 can be solved for the total maneuver time required for each axis denoted by $t_{f_{1}}, t_{f_{2}}, t_{f_{3}}$. For actual spacecraft with coupling between axes, the total maneuver time for all three axes should be identical; otherwise the boundary condition on slew angle and angular rate (zero for rest-to-rest maneuver) will not be satisfied for axes with smaller total maneuver time. The total maneuver time for all three axes is simply

$$
t_{f}=\max \left(t_{f_{1}}, t_{f_{2}}, t_{f_{3}}\right)
$$

This total maneuver time is used to modify the control profile to meet the slew angle and angular rate boundary conditions. A simple method is to modify the maximum torque required on each axis, which can be computed as

$$
u_{R}=\frac{B^{-1} J\left(\theta_{f}-\theta_{0}\right)}{\int_{0}^{t_{f}} \int_{0}^{\tau} f\left(\eta, t_{f}, \alpha\right) d \eta d \tau}
$$

It should satisfy the slew angle and angular rate constraints at the final time for all three axes when this modified control input, $u=u_{R} f\left(t, t_{f}, \alpha\right)$, is applied to Equation 2. The reference trajectories resulting from solving Equation 2 becomes

$$
\begin{aligned}
& \ddot{\theta}_{r e f}(t)=J^{-1} B u_{R} f\left(t, t_{f}, \alpha\right) \\
& \dot{\theta}_{r e f}(t)=\dot{\theta}_{0}+J^{-1} B u_{R} \int_{0}^{t} f\left(\tau, t_{f}, \alpha\right) d \tau \\
& \theta_{r e f}(t)=\theta_{0}+\dot{\theta}_{0} t+J^{-1} B u_{R} \int_{0}^{t} \int_{0}^{\tau} f\left(\eta, t_{f}, \alpha\right) d \eta d \tau
\end{aligned}
$$

Equation 8 has a common final time for all three axes whereas Equation 4 does not. The reference trajectories shown in Equation 8 are represented in the inertial frame. In order to convert the inertial frame reference into the spacecraft body frame where the control input is typically represented, the following relationship is used.

$$
\begin{aligned}
& \omega_{r e f}(t)=C\left(\theta_{r e f}\right) \dot{\theta}_{r e f} \\
& \dot{\omega}_{r e f}(t)=\frac{d}{d t}\left[C\left(\theta_{r e f}\right)\right] \dot{\theta}_{r e f}+C\left(\theta_{r e f}\right) \ddot{\theta}_{r e f}
\end{aligned}
$$

where, $C\left(\theta_{r e f}\right)$ transforms the inertial angular rate $\left(\dot{\theta}_{r e f}\right)$ into the body angular rate $\left(\omega_{\text {ref }}\right)$ with respect to the inertial frame. The reference control profile can be determined from rigid-body spacecraft model in Equation 1.

$$
u_{r e f}(t)=B^{-1}\left(J \dot{\omega}_{r e f}+\omega_{r e f} \times J \omega_{r e f}\right)
$$

The resulting reference control input is used as a feedforaward control to the spacecraft. This reference control profile satisfies rest-to-rest maneuver boundary constraints for rigid spacecraft with nonlinear coupling. However, when the flexibility is present in the spacecraft, residual vibrations will remain at the final time.

\section{INPUT SHAPING DESIGN FOR THREE-AXIS ROTATIONAL FLEXIBLE SPACECRFT}


It is previous shown in many literatures including Kim and Agrawal (2006) that input shaping techniques can improve the performance of slew maneuvers for single-axis flexible spacecraft simulator. With the input shaping technique, the reference control input is convoluted with a sequence of impulses to cancel vibrations from flexible modes. This is equivalent to cancelling the flexible poles by adding a timedelay which has zeros at the flexible pole locations. For a three dimensional rotational spacecraft, the equation of motion becomes nonlinear and it is unable to determine the frequency of the system (i.e. eigenvalues). Therefore, the system must be linearized to apply the input shaping technique. The equation of motion of the system can be linearized with respect to the final state of the system proposed by T. Singh, and S.R. Vadali (1993), which has shown to be effective. Equation of motion of a flexible spacecraft can be written as

$$
\begin{aligned}
& J \dot{\omega}+\omega \times J \omega+D^{T} \ddot{\eta}=B u \\
& \ddot{\eta}+\Gamma \dot{\eta}+\Lambda \eta+D \dot{\omega}=0
\end{aligned}
$$

where $J$ is the inertia matrix, $\omega$ is the angular rate of the spacecraft body, $D$ is a matrix representing rigid-elastic coupling, $\eta$ is the flexible state vector in generalized coordinates, $\Gamma$ is the diagonal damping matrix, and $\Lambda$ is a diagonal matrix representing the frequencies of the flexible body. Equation 11 is written in hybrid coordinates. The linearized equation of motion is simply written as

$$
\begin{aligned}
& J \dot{\omega}+D^{T} \ddot{\eta}=B u \\
& \ddot{\eta}+\Gamma \dot{\eta}+\Lambda \eta+D \dot{\omega}=0
\end{aligned}
$$

The system frequencies for input shaping technique are determined from Equation 12. The first step for reference control profile design is to determine the reference trajectories. Starting with the uncoupled rigid body equation, $J \ddot{\theta}=B u$, the reference trajectories can be similarly determined as shown as Equation 8 with a control input of $u=u_{R} f\left(t, t_{f}, \alpha\right)$. One option is to compute the reference input as shown in Equation 10 first, and then shape the resulting reference input. However, if the input shaper is directly used to shape the reference input in Equation 10, considerable rigid body position and velocity error will remain at the final time, which leads to the system states drifting away from the desired final state. Instead of shaping the reference input directly, the proposed development will shape the control input of $u=u_{R} f\left(t, t_{f}, \alpha\right)$. This ensures that the resulting reference trajectories will satisfy the boundary constraints of a slew maneuver. The shaped torque profile becomes

$$
u_{\text {shaped }}=\sum_{i=0}^{n} A_{i} \delta\left(t-t_{i}\right) * u_{R} f\left(t, t_{f}, \alpha\right)
$$

where $\sum_{i=0}^{n} A_{i} \delta\left(t-t_{i}\right)$ represents the impulse sequence for input shaping, and $*$ represents the convolution operation. When the reference trajectory is created with the shaped control input, uncoupled rigid body equation, $J \ddot{\theta}=B u$, can be used since it will still satisfy the constraints. However, it is more advantageous to use the linearized flexible spacecraft equation shown in Equation 12 to create a trajectory. The resulting reference trajectories from Equation 12 and Equation 13 should still satisfy the boundary constraints of a slew maneuver while more closely represent the actual spacecraft system than simple uncoupled system. In addition, the reference trajectories results from Equation 12 and Equation 13 will include flexible mode state $(\eta, \dot{\eta})$ trajectories. Using the same kinematic relationship as mentioned in the previous section,

$$
\begin{aligned}
& \omega_{r e f}(t)=C\left(\theta_{r e f}\right) \dot{\theta}_{r e f} \\
& \dot{\omega}_{r e f}(t)=\frac{d}{d t}\left[C\left(\theta_{r e f}\right)\right] \dot{\theta}_{r e f}+C\left(\theta_{r e f}\right) \ddot{\theta}_{r e f}
\end{aligned}
$$

the reference control profile including input shapers can be computed as

$$
u_{\text {ref }}(t)=B^{-1}\left(J \dot{\omega}_{\text {ref }}+\omega_{\text {ref }} \times J \omega_{\text {ref }}+D^{T} \ddot{\eta}\right)
$$

In the development of reference trajectories and control profile, linearization of the system model and recomputation of reference control from shaped control input will hinder from perfect cancellation of flexible modes. The robust version of the input shapers such as zero-vibrationderivative (ZVD) shapers are highly desirable in the design.

\section{INPUT SHAPING SIMULATION}

The mathematical model of the flexible spacecraft is shown in Equation 11. The parameters used for simulation are

$$
\begin{aligned}
& J=\left[\begin{array}{ccc}
3026 & 0 & 0 \\
0 & 440 & 0 \\
0 & 0 & 3164
\end{array}\right] \quad, \quad D=\left[\begin{array}{ccc}
-35.865 & -10.155 & 0 \\
0 & -5.255 & -35.372
\end{array}\right], \\
& \Lambda=\left[\begin{array}{cc}
1.112^{2} & 0 \\
0 & 0.885^{2}
\end{array}\right], \quad \Gamma=\left[\begin{array}{cc}
2(1.112) \zeta & 0 \\
0 & 2(0.885) \zeta
\end{array}\right], \quad \text { and }
\end{aligned}
$$

$\zeta=0.005$. The slew requirements are 30 degrees for all three axes from a zero attitude. The first step is to design rest-torest control profiles for the uncoupled rigid body problem. In order to exemplify the effect of flexibility, bang-bang control profile without smoothing is used in the simulation.
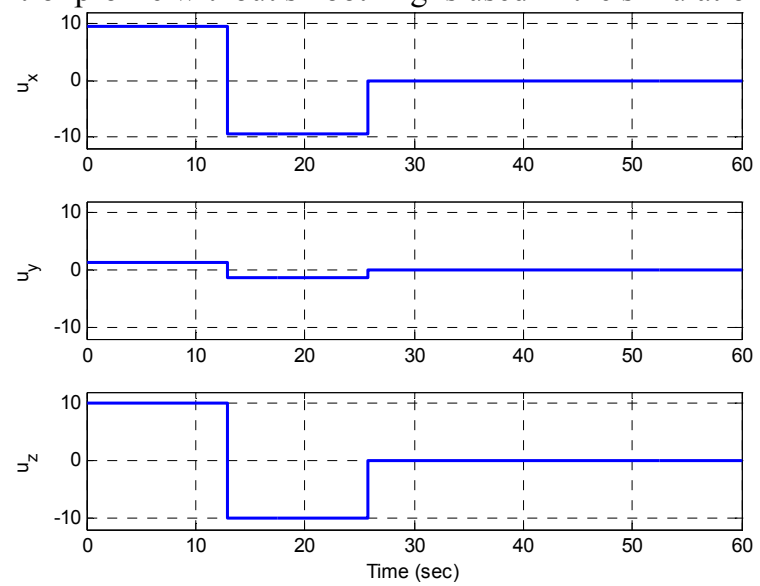

Figure 2: Bang-Bang Control for uncoupled axis rotation, $u=u_{R} f\left(t, t_{f}, \alpha\right), \alpha=0, t_{f}=25.742$ 
Figure 2 shows the bang-bang control profile for each axis when the final time is adjusted to have the same total maneuver time. When the rigid body spacecraft model is used, the reference control input is computed from Equation 10. Figure 3 shows the resulting reference control input when the rigid body spacecraft model is considered in the design. The control input is modified from Figure 2 to satisfy the 30 degree slew maneuvers for all three axes using Equation 10. When this reference control designed for rigid spacecraft is applied to the flexible spacecraft, considerable residual vibration will result as shown in Figure 4. The maximum amplitude of the residual vibration at the end of the maneuver is close to 1 degree from yaw angle trajectory in Figure 4.
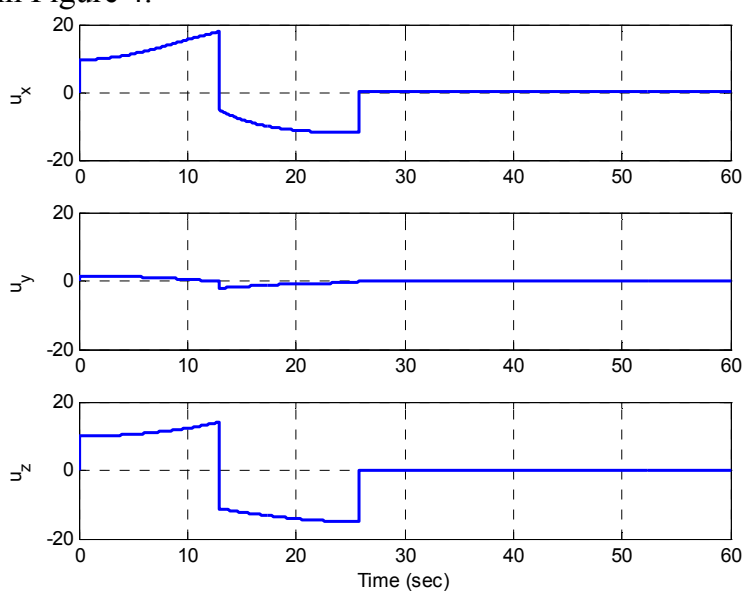

Figure 3: Reference Feedforward Control Profile Based on Rigid Body Spacecraft Model
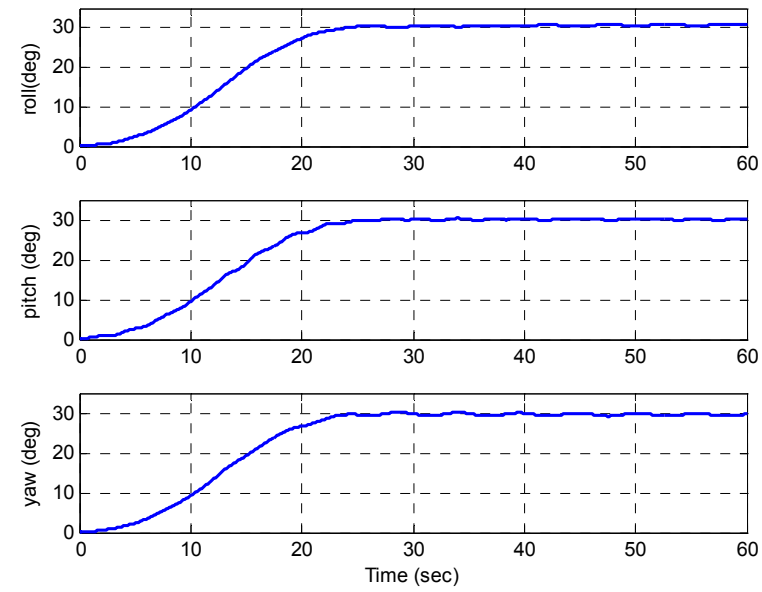

Figure 4: Resulting Trajectories with Reference Feedforward Control in Figure 3

Figure 5 shows the shaped input from Figure 2 using the frequencies of the system. The frequencies of the system will be different from the frequencies in $\Lambda$ matrix since the system frequency is computed from the whole system represented in Equation 11. The total maneuver time has increased as a result since each frequency in the system will add half of the damped period to the maneuver time. Figure 6 shows the reference control profile when the shaped bang- bang control in Figure 5 is modified to satisfy boundary conditions using Equation 15. The resulting trajectories showed good results with very little residual vibration (maximum residual vibration amplitude of 0.03 degree) and very little rigid body angular and angular rate errors.
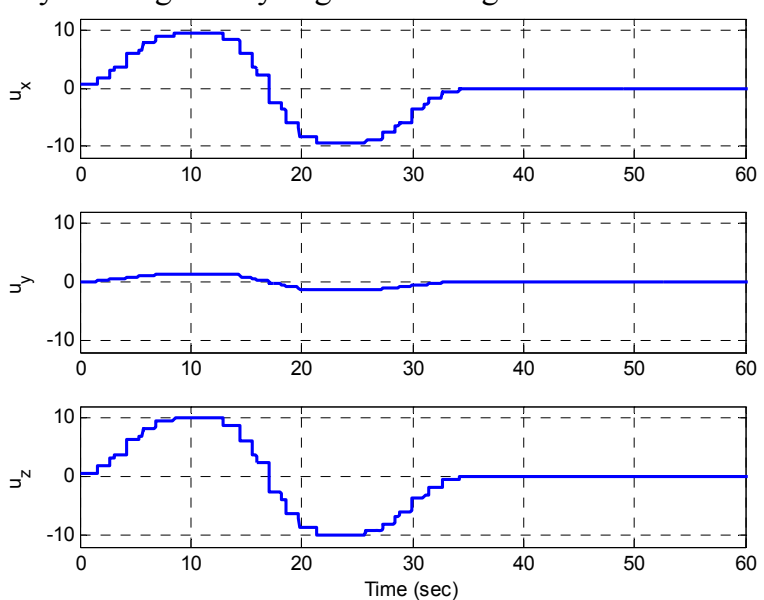

Figure 5: Input Shaped (ZVD) Bang-Bang Control Profile from Figure 2
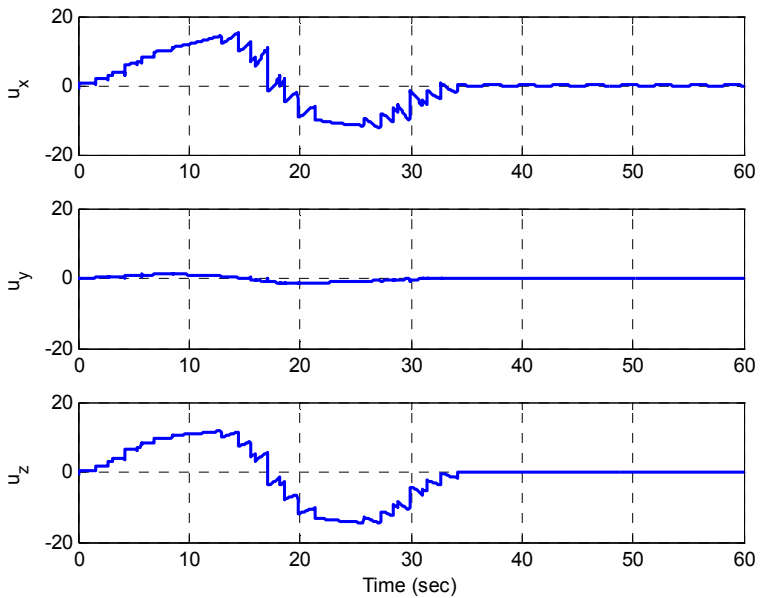

Figure 6: Reference Feedforward Control Profile with Input Shaping Based on Flexible Spacecraft Model
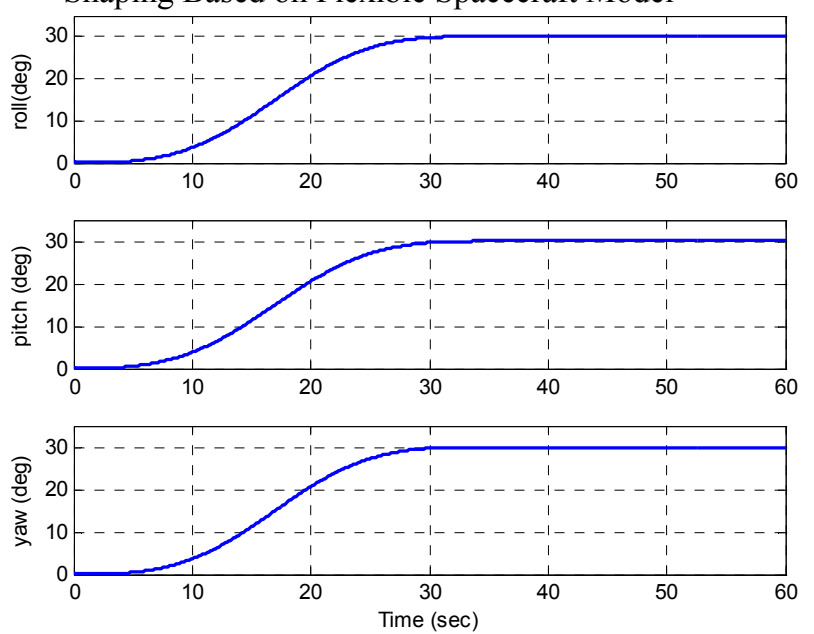

Figure 7: Resulting Trajectories with Reference Feedforward Control in Figure 6 


\section{EXPERIMENT WITH THE SPACECRAFT SIMULATOR}

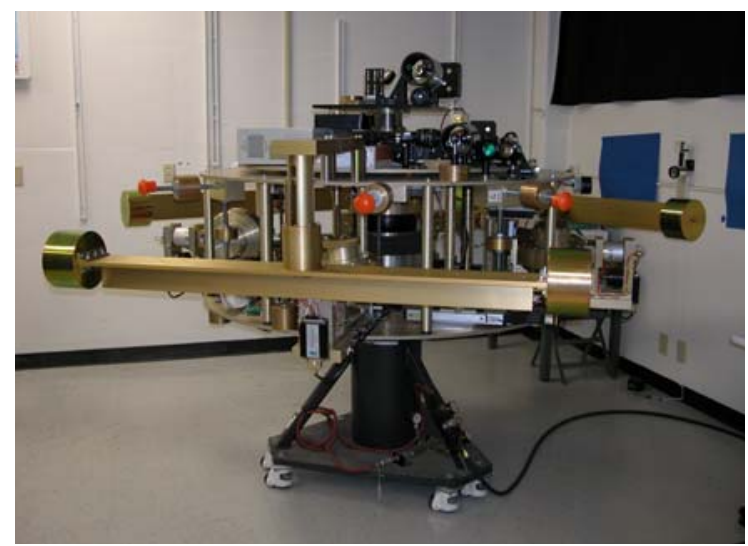

Figure 8: NPS Three-Axis Spacecraft Simulator 2

Figure 8 shows the current three-axis spacecraft simulator, which is an experimental test-bed for the Bifocal Relay Mirror Spacecraft. The key components of the spacecraft simulator include Spherical Air-Bearing, PC104 Onboard Computer with TCP/IP Ethernet Port, Serial Port, Analog I/Os, and Digital I/Os, Matlab/Simulink (via xPC Target) Real-Time Control Interface, IMU with integrated 3-Axis Fiber Optic Laser Gyros, Reconfigurable Control Moment Gyroscope (CMG) Array, a IR sensor, a Magnetometer and 2 inclinometers, an Automatic Mass Balancing System, and Passive Safety bumpers. Three flexible mass simulators are also installed on the test-bed, which provide low frequency vibration $(\sim 0.1 \mathrm{~Hz})$ for all three axes and comprise about $9 \%$ of the principal moment of inertia. A detailed drawing of each flexible mass is shown in Figure 11. An adjustable length torsional rod provides stiffness for the flexible masses. The flexible masses can be securely locked to simulate rigid body spacecraft when desired. Before performing slew maneuver experiments, system identification of the spacecraft should be performed. When the flexible mass simulators are locked in places, the rigid spacecraft has an inertia matrix of

$$
J=\left[\begin{array}{ccc}
287.58 & -40.25 & 2.91 \\
-40.25 & 262.70 & -19.09 \\
2.91 & -19.09 & 427.65
\end{array}\right]
$$

The value of the inertia matrix is determined using least square method using momentum balance equation. It has been assumed that the rigid-elastic coupling is zero simply because it is currently not available. All three flexible masses have frequencies very close to $0.1 \mathrm{~Hz}$ with damping values of around $1 \%$. Applying these values into Equation 11 provides the mathematical model of the current spacecraft simulator. Because we ignored the rigid-elastic coupling, it is expected that additional error will be present for slew maneuver control. Initial attitude is $\left[\begin{array}{lll}0 & 0 & 0\end{array}\right]^{T}$ degrees and the final attitude is $\left[\begin{array}{lll}0 & 0 & 17\end{array}\right]^{T}$ degrees for slew maneuver experiments. First step for slew control design is to determine control profile and trajectories for a simple uncoupled system. In the current spacecraft test-bed, three Control Moment Gyroscopes (CMGs) are used as primary actuators. This CMG array will exhibit a singularity state for certain geometric configurations of gimbal angles. For an agile maneuver, it is highly desirable to utilize the full momentum envelop of the CMG array without encountering singularity problems. The CMG steering law is written as

$$
\dot{h}=A(\delta) \dot{\delta} \rightarrow \dot{\delta}=A(\delta)^{-1} \dot{h}
$$

where $\dot{h}$ is the torque from the CMG array, $\dot{\delta}$ is the gimbal rate vector, and $A(\delta)$ is the Jacobian matrix determining the gimbal steering law. The singularity condition corresponds to the case where the matrix $A(\delta)$ becomes singular. When the matrix $A(\delta)$ is singular, the gimbal rate cannot be determined from the amount of torque required. In addition, the maximum control torque from the CMG will be limited by the gimbal rate such that

$$
\dot{h}_{\max }=A(\delta) \dot{\delta}_{\text {max }}
$$

Since the matrix $A(\delta)$ is time-varying, the maximum available torque is not constant and Equation 3 cannot be used. Instead of using Equation 3 to determine the control torque for uncoupled system, simple parameter optimization routine is formulated to determine the gimbal rate which satisfies the slew boundary conditions. Gimbal rates of three CMGs are parameterized as versine profiles. The uncoupled system used for optimization ignores the flexibility and the cross coupling.

$$
J_{d} \dot{\omega}=-A(\delta) \dot{\delta}-\omega \times \mathrm{h}(\delta)
$$

It is noted that the non-diagonal elements of the inertia matrix is also ignored to formulate a simpler problem. The resulting gimbal rate profile which satisfies the constraints are shown in Figure 9. The maximum gimbal rate is limited by $0.5 \mathrm{rad} / \mathrm{s}$. The gimbal rate cannot be parameterized as bang-bang in real systems since CMG system cannot change the rate instantaneously. Therefore, versine profile is used instead.
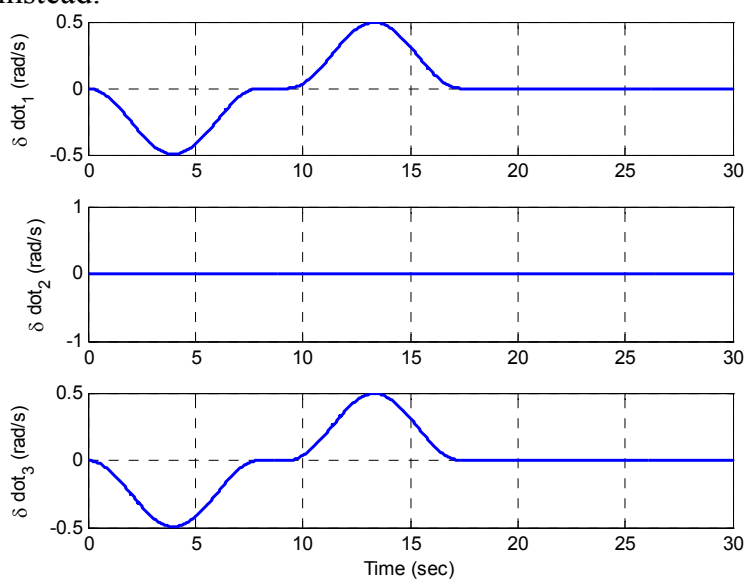

Figure 9: Gimbal Rate Profile for Uncoupled System

The corresponding control torque profile for Figure 9 is shown in Figure 10. This control profile is used to create 
reference trajectories of the system. When only the rigid body is considered, the reference control torque profile is computed as shown in Figure 11. In order to apply this control to our spacecraft simulator with CMGs, the required torque should be converted into a required gimbal rate command. Figure 12 shows the required gimbal rate when steering law in Equation 16 is used. At around 4 seconds in Figure 12, gimbal rate command showed a very large value which indicates that the system is singular.
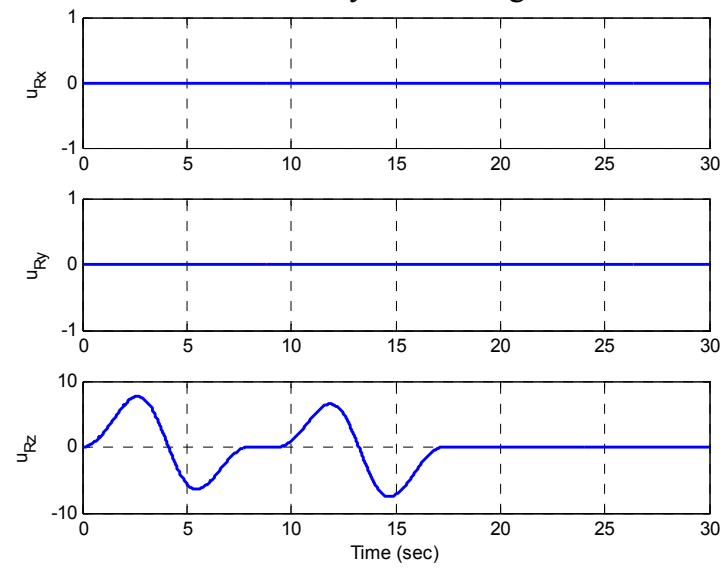

Figure 10: Control Torque for Uncoupled System
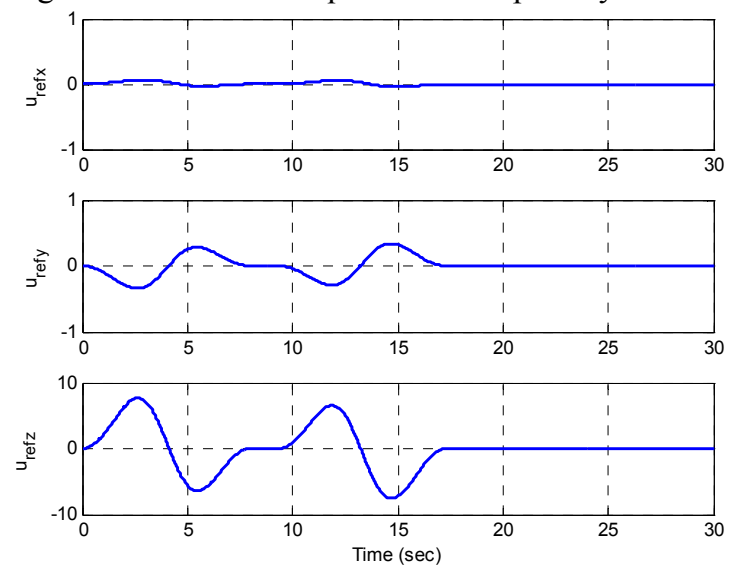

Figure 11: Reference Control Torque Based on Rigid Body Spacecraft Model

Therefore, this gimbal rate command cannot be used in its current form. In fact, when the optimization problem is solved to determine the gimbal rate profile in Figure 9, the gimbal trajectories also undergoes singular states. However, no inversion is necessary to solve the optimization problem, and smooth control profile can be obtained. On the other hand, solving for gimbal rate from the control torque cannot be simply determined. For experiments, gimbal rate for uncoupled system will be used for residual vibration demonstration purpose. Figure 13 shows input shaped control profiles using ZVD at $0.1 \mathrm{~Hz}$ from the control torque in Figure 10. The slew trajectories were generated and the resulting control torque profile is shown in Figure 14. Luckily, when the reference control torque profile shown in Figure 14 is used to determine the reference gimbal rate profiles, no singularity has encountered. This is due to the fact that the amplitude of the control has decreased as a result of input shaping and the gimbal system stayed within the singularity-free momentum space. The resulting reference gimbal rate profile with input shaping is shown in Figure 15.
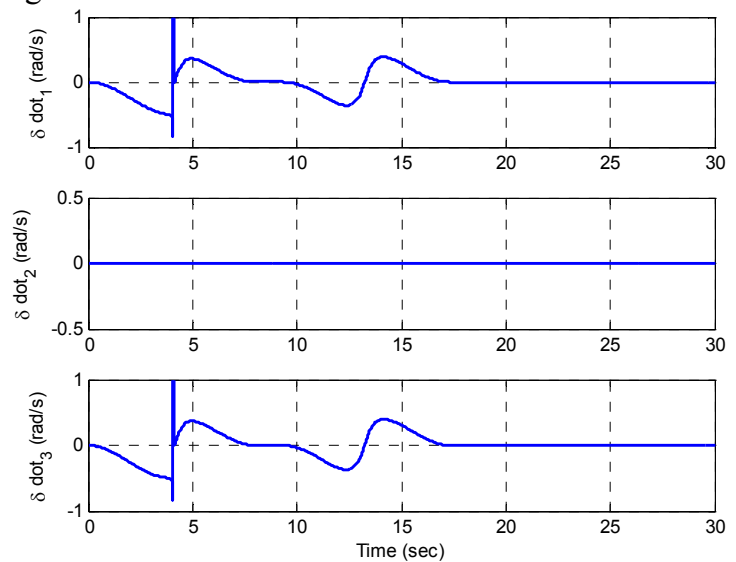

Figure 12: Gimbal Rate Profile for Generating Control Torque in Figure 11
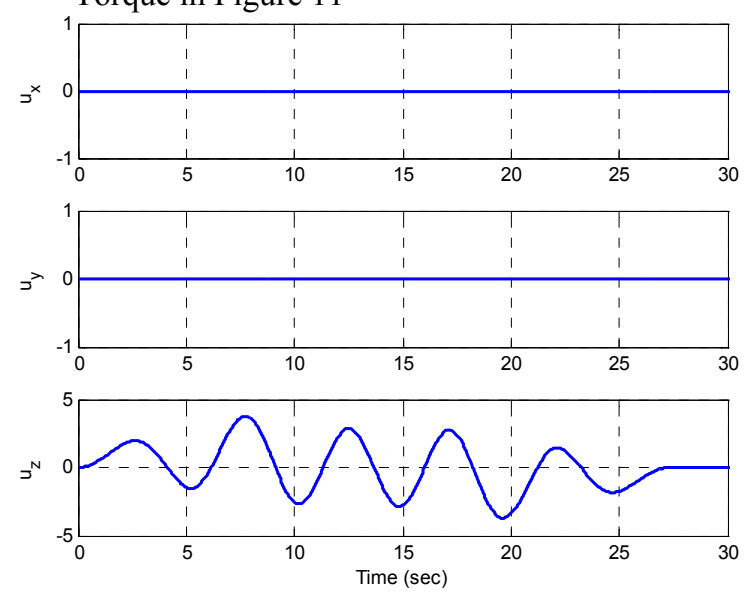

Figure 13: Input Shaped Control Profile using Control Torque Profile shown in Figure 10
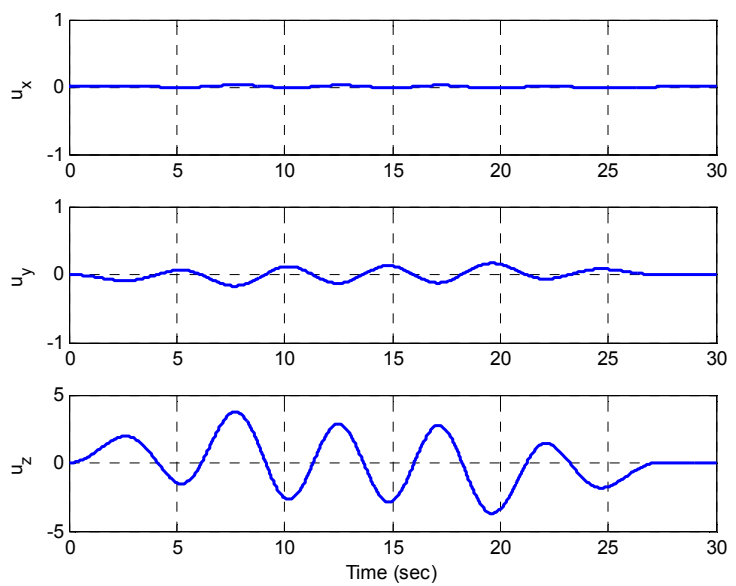

Figure 14: Reference Control Torque Profile with Input Shaping 

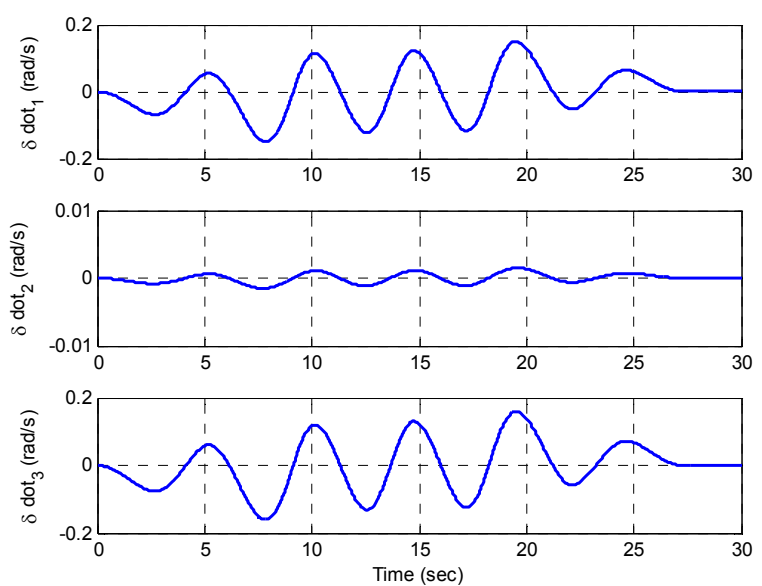

Figure 15: Gimbal Rate Profile with Input Shaping

For comparison purposes, two different control profiles shown in Figure 9 and Figure 15 are applied to the spacecraft simulator. Only feedforward control is applied because feedback control may create singularity problems near singular states. In order to close a feedback loop about the reference trajectories, the control profile could be redesigned to stay within the singularity-free region. However, with small usage of momentum space, maneuver will be slow and flexibility and non-linear effect will not be exemplified
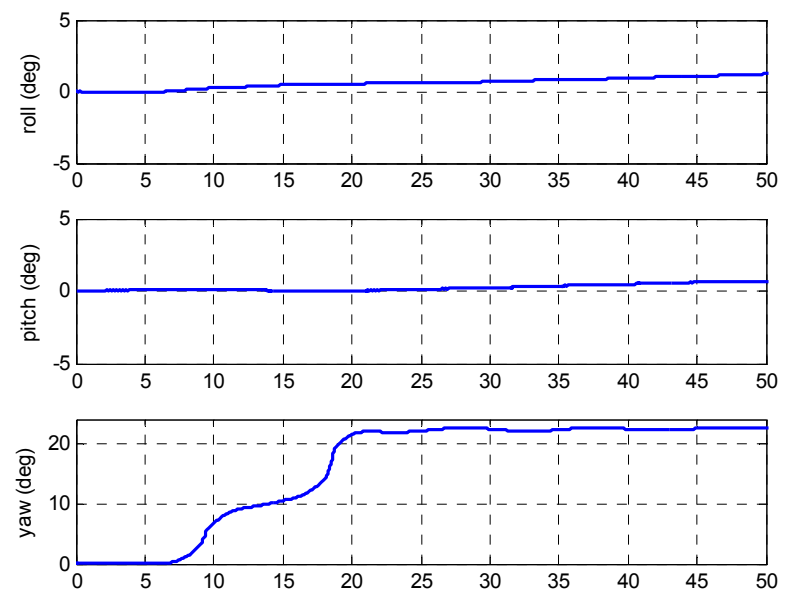

Figure 16: Spacecraft Simulator Attitude Trajectories with Gimbal Rate shown in Figure 9

Figure 16 Shows the attitude trajectory of the spacecraft simulator when the gimbal rate shown in Figure 9 is applied to the experimental test-bed. It showed a residual vibration (maximum amplitude of around $1 \mathrm{deg}$ ) at the end of the maneuver. Figure 17 shows the results when the gimbal rate shown in Figure 15 is applied. Even though the rigid-elastic coupling is ignored in the spacecraft model, the residual vibration is almost completely eliminated. Figure 16 showed a large steady-state angular error in yaw angle since the trajectories are based on the rigid spacecraft. Figure 17 shows some residual rigid body angular rate in yaw angle due to zero elastic-rigid coupling assumption.
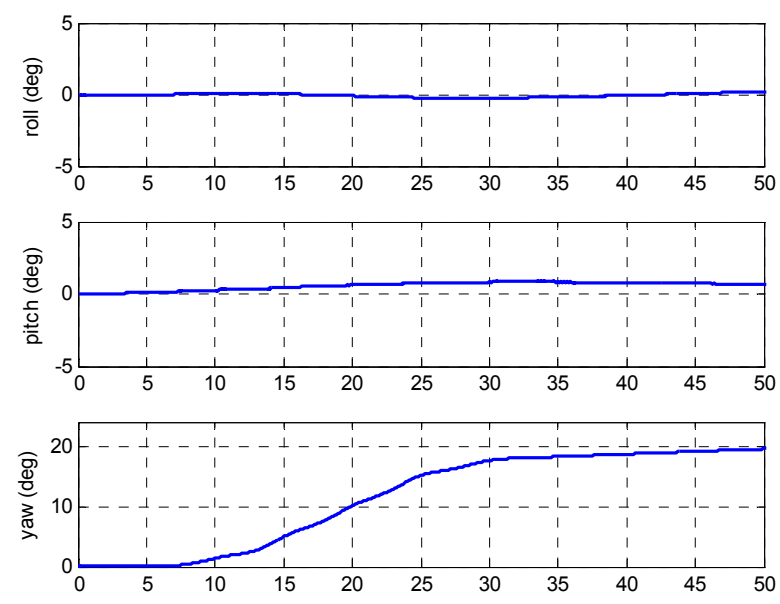

Figure 17: Spacecraft Simulator Attitude Trajectories with Gimbal Rate shown in Figure 15

\section{CONCLUSION}

In this paper, input shaping technique is applied to the flexible nonlinear spacecraft problems. First control profile is designed by ignoring nonlinear and flexible terms in the equation of motion. This control input is used for input shaping. The resulting shaped input is further modified to include the nonlinear and coupling effect. It has shown in the simulation and experiments that this method can be effective in designing slew trajectories for flexible spacecraft. Further study is necessary to compare performances with other leading alternative techniques for slew maneuvers and complete demonstration of the techniques using the spacecraft simulator.

\section{REFERENCES}

B. Wie, D. Bailey. and C. Heiberg (2001), Singularity Robust Steering Logic for Redundant Single-Gimbal Control moment Gyros, Journal of Guidance Control and Dynamics, Vol. 24, No. 5. September-October.

T. Singh, and S.R. Vadali (1993), Input Shaped Control of Three-Dimensional Maneuvers of Flexible Spacecraft, Journal of Guidance Control, and Dynamics, Vol. 16, No. 6.

M. J. Bell and J.L. Junkins (1994), Near Minimum-Time Three Dimensional Maneuvers of Rigid and Flexible Spacecraft, Journal of the Astronautical Sciences, Vol. 42., No. 4.

G. Marguiles and Aubrun (1978), Geometric Theory of Single Gimbal Control Moment Gyro Systems, Journal of the Astronautical Sciences, Vol. XXVI, No. 2.

T. Singh and W. Singhose (2002), Tutorial on Input Shaping/Time Delay Control of Maneuvering Flexible Structures, 2002 American Control Conference, May 810, Anchorage, Alaska.

J. J. Kim and B. N. Agrawal (2006), Experiments on Jerk Limited Slew Maneuvers of a Flexible Spacecraft, AIAA Guidance, Navigation, Control Conference, Keystone, CO. 